\title{
EXTENSIONS OF HOLOMORPHIC MAPS
}

\author{
BY
}

\section{PETER KIERNAN}

ABSTRACT. Several generalizations of the big Picard theorem are obtained. We consider holomorphic maps $f$ from $X-A$ into $M \subset Y$. Under various assumptions on $X, A$, and $M$ we show that $f$ can be extended to a holomorphic or meromorphic map of $X$ into $Y$.

1. Introduction. The big Picard theorem says that any holomorphic map $f$ from the punctured disk $D^{*}$ into the Riemann sphere $P_{1}(C)$ which omits three points can be extended to a holomorphic map $f: D \rightarrow P_{1}(C)$. Kobayashi [3] has obtained the following generalization of this. Let $A$ be a closed submanifold of the complex manifold $X$ and let $M$ be a hyperbolically imbedded(1) subspace (definition below) of the complex space $Y$. Then any holomorphic map $f: X-A \rightarrow M$ can be extended to a holomorphic map $f: X \rightarrow Y$. The purpose of this paper is to consider further generalizations of this result. In particular, it will be shown that if $A$ has singularities, then in general $f$ can only be extended to a meromorphic map. However, if the singularities of $A$ are normal crossings, then $f$ can be extended to a holomorphic map into $Y$.

2. Definitions. Our main tool for investigating the extension of holomorphic maps will be the Kobayashi pseudo-distance $d_{M}$ which is associated with the complex space $M$. For completeness, we shall give the definition and a summary of some of the main properties of $d_{M}$ which we use. The reader can consult [3] for further details.

Let $p$ and $q$ be points in the complex space $M$. By a chain $\alpha$ from $p$ to $q$, we mean a sequence of points $p=p_{0}, p_{1}, \cdots, p_{k}=q$ in $M$, points $a_{1}, \cdots, a_{k}$ in the unit disk $D=\{z \in \mathbf{C}|| z \mid<1\}$ and holomorphic maps $f_{1}, \cdots, f_{k}$ of $D$ into $M$ with $f_{i}(0)=p_{i-1}$ and $f_{i}\left(a_{i}\right)=p_{i}$. The length $|\alpha|$ of $\alpha$ is defined by

$$
|\alpha|=\sum_{i=1}^{k} d\left(0, a_{i}\right)=\sum_{i=1}^{k} \log \frac{1+\left|a_{i}\right|}{1-\left|a_{i}\right|}
$$

Received by the editors February 1, 1971 and, in revised form, October 20, 1971. AMS (MOS) subject classifications (1970). Primary 32H25, 32H 20.

Key words and phrases. Big Picard theorem, hyperbolic, Kobayashi pseudo-distance, Satake compactification, symmetric bounded domain.

(1) Several characterizations of hyperbolically imbedded spaces and another application of Theorem 2 are given in a recent paper by this author. The paper is entitled Hyperbolically imbedded spaces and the big Picard theorem and has been submitted to Mathematische Annalen. 
where $d$ is the Poincaré-Bergman distance on $D$. It is given by the metric

$$
d s^{2}=\frac{1}{\left(1-|z|^{2}\right)^{2}} d z d \bar{z}
$$

We set $d_{M}(p, q)=\inf _{a \in A}|\alpha|$, where $A$ is the set of all chains from $p$ to $q$. It is easy to see that $d_{M}$ is a pseudo-distance on $M$. If $d_{M}$ is a (complete) metric, we say that $M$ is (complete) byperbolic.

Some of the properties of this pseudo-distance which shall be useful to us are

(1) $d_{D}$ is the Poincare-Bergman distance on the unit disk $D$.

(2) Let $D^{*}=\{z \in \mathbf{C}|0<| z \mid<1\}$. Then $d_{D^{*}}$ is a complete metric corresponding to the hermitian metric

$$
d s^{2}=\frac{1}{|z|^{2}\left(\log |z|^{2}\right)^{2}} d z d \bar{z} .
$$

Thus $D^{*}$ is complete hyperbolic. However, if $\gamma_{r}=\left\{z=r e^{i t} \mid 0 \leq t \leq 2 \pi\right\}$, then the diameter of $\gamma_{r}$ converges to 0 as $r$ converges to 0 .

(3) If $f: M \rightarrow N$ is holomorphic, then $f$ is distance decreasing. That is, if $p, q \in M$ then $d_{N}(f(p), f(q)) \leq d_{M}(p, q)$.

(4) $M$ is (complete) hyperbolic iff the universal covering space of $M$ is (complete) hyperbolic.

Let $Y$ be a complex space and let $M$ be a relatively compact, hyperbolic complex subspace of $Y$. We say that $M$ is byperbolically imbedded in $Y$ if either of the following (equivalent) conditions are satisfied:

(i) If $p$ and $q$ are boundary points of $M$ and if $\left\{p_{n}\right\}$ and $\left\{q_{n}\right\}$ are sequences in $M$ such that $p_{n} \rightarrow p, q_{n} \rightarrow q$, and $d_{M}\left(p_{n}, q_{n}\right) \rightarrow 0$, then $p=q$.

(ii) If $p$ is a boundary point of $M$ and $U$ is a neighborhood of $p$ in $Y$, there exists a neighborhood $V$ of $p$ in $Y$ such that $\bar{V} \subset U$ and the distance between $M \cap(Y-U)$ and $M \cap V$ with respect to $d_{M}$ is positive.

Intuitively, a relatively compact subspace $M$ is hyperbolically imbedded in $Y$ if $M$ is hyperbolic and if the "distance" between any two distinct boundary points is greater than zero.

The following are some examples of hyperbolically imbedded spaces.

(5) Let $M$ be a compact hyperbolic manifold. Then $M$ is hyperbolically imbedded in $Y=M$.

(6) Let $Y=P_{1}(\mathrm{C})$ and $M=P_{1}(\mathrm{C})$ - \{three points $\}$. $M$ is covered by the unit disk and therefore it is complete hyperbolic. Since $M$ has only isolated boundary points, $M$ is hyperbolically imbedded in $Y$.

(7) If $M_{1}$ and $M_{2}$ are hyperbolically imbedded in $Y_{1}$ and $Y_{2}$ respectively, then $M_{1} \times M_{2}$ is hyperbolically imbedded in $Y_{1} \times Y_{2}$. To see this let $\pi_{j}: M_{1} \times$ $M_{2} \rightarrow M_{j}$ be the projection onto $M_{j}$ and let $p=\left(p^{\prime}, p^{\prime \prime}\right), q=\left(q^{\prime}, q^{\prime \prime}\right)$, $\left\{p_{n}=\left(p_{n}^{\prime}, p_{n}^{\prime \prime}\right)\right\}$, and $\left\{q_{n}=\left(q_{n}^{\prime}, q_{n}^{\prime \prime}\right)\right\}$ be as in (i) above. If $d_{M_{1} \times M_{2}}\left(p_{n}, q_{n}\right) \rightarrow 0$, then $d_{M_{1}}\left(p_{n}^{\prime}, q_{n}^{\prime}\right) \rightarrow 0$ and $d_{M_{2}}\left(p_{n}^{\prime \prime}, q_{n}^{\prime \prime}\right) \rightarrow 0$ since $\pi_{1}$ and $\pi_{2}$ are distance de- 
creasing. Thus $p^{\prime}=q^{\prime}$ and $p^{\prime \prime}=q^{\prime \prime}$ and therefore $M_{1} \times M_{2}$ is hyperbolically imbedded in $Y_{1} \times Y_{2}$.

(8) Let $P_{2}(\mathrm{C})$ be the 2-dimensional complex projective space and let $Q$ be a complete quadrilateral. That is, $Q$ is the union of the six projective lines which pass through a set of four points in general position. Then $M=P_{2}(C)-Q$ is hyperbolically imbedded in $P_{2}(\mathrm{C})$; see [3, p. 92].

Finally, we recall the definition of meromorphic map given by Remmert in [6]. A meromorphic mapping $f$ from a complex space $X$ into a complex space $Y$ is a correspondence satisfying

(i) For each point $x \in X, f(x)$ is a nonempty compact subset of $Y$.

(ii) The graph $\Gamma_{f}=\{(x, y) \in X \times Y \mid y \in f(x)\}$ of $f$ is a connected complex subspace of $X \times Y$ with $\operatorname{dim} \Gamma_{f}=\operatorname{dim} X$.

(iii) There exists a dense subset $X^{\prime}$ of $X$ such that $f(x)$ is a single point for each $x$ in $X^{\prime}$. Remmert has shown that the codimension of the set $X-X^{\prime}$ is $\geq 2$. Thus if $X$ is a normal space and $\operatorname{dim} X=1$, then $f$ is actually holomorphic.

3. Extension theorems. The following theorem is the basis for our extension theorems. The proof is essentially the same as Mrs. Kwack's proof of Theorem 3 in [5], although our statement is slightly more general. The winding number argument used below is due to Grauert and Reckziegel.

Theorem 1. Let $M$ be byperbolically imbedded in $Y$ and let $f_{k}: D^{*} \rightarrow M$ be a sequence of holomorphic maps where $D^{*}=\{z \in \mathrm{C}|0<| z \mid<1\}$. Let $\left\{z_{k}\right\}$ and $\left\{z_{k}^{\prime}\right\}$ be sequences in $D^{*}$ converging to 0 and such that $f_{k}\left(z_{k}^{\prime}\right) \rightarrow q \in Y$. Then

(i) $f_{k}\left(z_{k}\right) \rightarrow q$.

(ii) Any bolomorpbic map $f: D^{*} \rightarrow M$ extends to a bolomorphic map $f: D \rightarrow Y$.

(iii) $f_{k}(0) \rightarrow q$.

Proof. (i) Assume that $f_{k}\left(z_{k}\right) \rightarrow p \neq q$ and that $\left|z_{k}\right| \leq\left|z_{k}^{\prime}\right|$. Choose a coordinate neighborhood $U$ of $p$ such that $U$ is an analytic subset of $W=\left\{\left.\left(w_{1}, \cdots, w_{n}\right) \in \mathrm{C}^{n}|| w_{1}\right|^{2}+\cdots+\right.$ $\left.\left|w_{n}\right|^{2}<2\right\}, q \notin U$, and $p=(0, \cdots, 0)$. Let $\rho_{k}(t)=z_{k} e^{i t}$ for $0 \leq t \leq 2 \pi$. Then the diameters of the sets $\rho_{k}$ with respect to $d_{D^{*}}$ are converging to 0 . Since $M$ is hyperbolically imbedded in $Y$ and since $f_{k}$ is distance decreasing, this implies $f_{k}\left(\rho_{k}\right)$ is converging to $p$. Thus for all sufficiently large $k$, there exists an annulus $R_{k}^{\prime}$ centered at 0 such that $\rho_{k} \subset R_{k}^{\prime}$ and $f_{k}\left(R_{k}^{\prime}\right) \subset B=\left\{\left(w_{1}, \cdots, w_{n}\right)\right\}$ $\left.\left|w_{1}\right|^{2}+\cdots+\left|w_{n}\right|^{2}<1\right\}$. Let $R_{k}$ be the largest such annulus. We can assume that either $R_{k}$ is not a punctured disk for any $k$ or that $R_{k}$ is a punctured disk for all $k$. We consider the former case first. Since $f_{k}\left(z_{k}^{\prime}\right) \rightarrow q \notin U$, there exist $a_{k}$ and $b_{k}$ in $\vec{R}_{k}$ with $\left|a_{k}\right|<\left|b_{k}\right|$ and $\left|f_{k}\left(a_{k}\right)\right|=\left|f_{k}\left(b_{k}\right)\right|=1$. By taking a subsequence and relabelling, we may assume that $f_{k}\left(a_{k}\right) \rightarrow q^{\prime} \in \bar{B}$ and $f_{k}\left(b_{k}\right) \rightarrow q^{\prime \prime} \in \vec{B}$. Let $\sigma_{k}$ and $\tau_{k}$ be the curves defined by $\sigma_{k}(t)=a_{k} e^{i t}$ and $\tau_{k}(t)=b_{k} e^{i t}$ for $0 \leq t \leq 2 \pi$. By the same reasoning used for $\rho_{k}$, we see that $f_{b}\left(\sigma_{b}\right) \rightarrow q$ ' and $f_{k}\left(\tau_{k}\right) \rightarrow q:$. 
Let $f_{k}=\left(f_{k}^{1}, \cdots, f_{k}^{n}\right)$ in $f_{k}^{-1}(U)$. By rotating the coordinate ball $W$ if necessary, we see that the winding number of the curves $f_{k}^{1}\left(\sigma_{k}\right)$ and $f_{k}^{1}\left(\tau_{k}\right)$ around the point $f_{k}^{1}\left(z_{k}\right)$ is 0 for all $k$ sufficiently large. By Cauchy's theorem, we have

$$
\int_{\sigma_{k}} \frac{f_{k}^{1 \prime}(z)}{f_{k}^{1}(z)-f_{k}^{1}\left(z_{k}\right)} d z=\int_{f_{k}^{1}\left(\sigma_{k}\right)} \frac{d w_{1}}{w_{1}-f_{k}^{1}\left(z_{k}\right)}=0
$$

and

$$
\int_{\tau_{k}} \frac{f_{k}^{1 \prime}(z)}{f_{k}^{1}(z)-f_{k}^{1}\left(z_{k}\right)} d z=\int_{f_{k}^{1}\left(\tau_{k}\right)} \frac{d w_{1}}{w_{1}-f_{k}^{1}\left(z_{k}\right)}=0
$$

Thus

$$
0=\int_{\sigma_{k}} \frac{f_{k}^{1 \prime}(z)}{f_{k}^{1}(z)-f_{k}^{1}\left(z_{k}\right)} d z-\int_{\tau_{k}} \frac{f_{k}^{1 \prime}(z)}{f_{k}^{1}(z)-f_{k}^{1}\left(z_{k}\right)} d z=2 \pi i(P-N)
$$

where $N$ and $P$ are the number of zeros and poles of the function $f_{k}^{1}(z)-f_{k}^{1}\left(z_{k}\right)$ on the annulus $R_{k^{*}}$. This is a contradiction since $N>0$ and $P=0$.

If $R_{k}$ is a punctured disk for all $k$, then the boundary of $\bar{R}_{k}$ is $\tau_{k}$ where $b_{k}$ and $\tau_{k}$ are as above. Since $f_{k}\left(R_{k}\right) \subset B, f_{k}$ extends holomorphically to $\bar{R}_{k}$ with $f_{k}(0)$ in $B$. Setting $\sigma_{k}=\varnothing$, the argument used in the preceding paragraph leads to a contradiction. This proves (i).

(ii) Let $\left\{z_{k}^{\prime}\right\}$ be any sequence in $D^{*}$ such that $f\left(z_{k}^{\prime}\right) \rightarrow q \in Y$. Define $f(0)=$ q. By (i), $f$ is continuous. The Riemann extension theorem now implies that $f$ is holomorphic at 0 .

(iii) If $f_{k}(0)$ does not converge to $q$, then without loss of generality we may assume that $f_{k}(0) \rightarrow p \neq q$. Since each $f_{k}$ is continuous, there exists a sequence $\left\{z_{k}\right\}$ in $D^{*}$ such that $z_{k} \rightarrow 0$ and $f_{k}\left(z_{k}\right) \rightarrow p$. This contradicts (i). Q.E.D.

The following theorem was proven by $\mathrm{Kwack}$ in the case when $M$ is compact and by Kobayashi in the case when $A$ is nonsingular.

Theorem 2. Let $A$ be a closed complex subspace of a (nonsingular) complex manifold $X$. If the singularities of $A$ are normal crossings and if $M$ is byperbolically imbedded in $Y$, then any bolomorphic map $f: X-A \rightarrow M$ extends to a bolomorphic map $f: X \rightarrow Y$.

Proof. Since the singularities of $A$ are normal crossings, we can assume that

$$
X=D \times \cdots \times D=D^{n} \times D^{l}=\left\{\left(w_{1}, \cdots, w_{n}, t_{1}, \cdots, t_{l}\right)|| w_{i} \mid<1 \text { and }\left|t_{j}\right|<1\right\}
$$

and that

$$
X-A=\left(D^{*}\right)^{n} \times D^{l}=\left\{\left(w_{1}, \cdots, w_{n}, t_{1}, \cdots, t_{l}\right)|0<| w_{i} \mid<1 \text { and }\left|t_{j}\right|<1\right\} .
$$

The proof is by induction on $n$. 
Case 1. $X-A=D^{*}$. This is part (ii) of Theorem 1 .

Case 2. Assume we can extend $f$ when $X-A=\left(D^{*}\right)^{n}$. We show that this implies we can extend $f$ if $X-A=\left(D^{*}\right)^{n} \times D^{l}$.

Let $w=\left(w_{1}, \cdots, w_{n}\right) \in D^{n}$ and $t=\left(t_{1}, \cdots, t_{l}\right) \in D^{l}$. We denote the point $\left(w_{1}, \cdots, w_{n^{\prime}} t_{1}, \cdots, t_{l}\right) \in D^{n} \times D^{l}$ by $(w, t)$. Let $f:\left(D^{*}\right)^{n} \times D^{l} \rightarrow M$ be holomorphic and define $f_{t}:\left(D^{*}\right)^{n} \rightarrow M$ by $f_{t}(w)=f(w, t)$. Since each map $f_{t}$ extends to $D^{n}$, we can extend $f$ to a map from $D^{n} \times D^{l}$ into $Y$ by defining $f(w, t)=f_{t}(w)$. By the Riemann extension theorem, it suffices to show that the extended map $f$ is continuous.

We assume that $f$ is not continuous at some point, say $(w, 0)$ for simplicity, and then obtain a contradiction. If $f$ is not continuous at $(w, 0)$, then there exists a sequence of points $\left(w^{k}, t^{k}\right)$ in $\left(D^{*}\right)^{n} \times\left(D^{*}\right)^{l}$ with $\left(u^{k}, t^{k}\right) \rightarrow(w, 0)$ and such that the sequence $f\left(w^{k}, t^{k}\right) \rightarrow q \neq f(w, 0)$. Define $f_{k}: D^{*} \rightarrow M$ by $f_{k}(z)=$ $f\left(w^{k}, z t^{k} /\left|t^{k}\right|\right)$. Let $z_{k}^{\prime}=\left|t^{k}\right|$. Since $z_{k}^{\prime} \rightarrow 0$ and $f_{k}\left(z_{k}^{\prime}\right)=f\left(w^{k}, t^{k}\right) \rightarrow q$, part (iii) of Theorem 1 implies that $f_{k}(0)=f\left(w^{k}, 0\right) \rightarrow q$. But $f_{t}$ is continuous for each $t$ and therefore $f\left(w^{k}, 0\right) \rightarrow f(w, 0) \neq q$. Thus the assumption that $f$ is not continuous is false.

Case 3. Assume that $f$ can be extended if $X-A=\left(D^{*}\right)^{n} \times D^{l}$. We show that this implies that $f$ can be extended if $X-A=\left(D^{*}\right)^{n+1}$. By the inductive hypothesis, we can extend $f$ to $D^{n+1}-\{(0, \ldots, 0)\}$. The map $g: D^{*} \rightarrow M$ defined by $g(z)=f(z, \cdots, z)$ extends to $D$. Define $f(0, \cdots, 0)=g(0)$. It suffices to show that $f$ is continuous.

If $f$ is not continuous, there exists a sequence $\left(w^{k}, t^{k}\right)=\left(w_{1}^{k}, \ldots, w_{n}^{k}, t^{k}\right)$ in $\left(D^{*}\right)^{n+1}$ such that $f\left(w^{k}, t^{k}\right) \rightarrow q \neq f(0, \cdots, 0)$. By applying Theorem 1 with $f_{k}(z)=f\left(z w^{k} /\left|w^{k}\right|, t^{k}\right)$ and $z_{k}^{\prime}=\left|w^{k}\right|$, we see that $f\left(0, t^{k}\right)=f_{k}(0) \rightarrow q$. On the other hand, if we apply Theorem 1 with

$$
f_{k}(z)=f\left(z t^{k} /\left|t^{k}\right|, \cdots, z t^{k} /\left|t^{k}\right|, t^{k}\right) \text { and } \quad z_{k}^{\prime}=\left|t^{k}\right|
$$

we see that $f\left(0, t^{k}\right) \rightarrow f(0, \cdots, 0) \neq q$. This is a contradiction and therefore $f$ extends holomorphically. Q.E.D.

The following example shows that if $M$ is not compact, restrictions on the singularities of $A$ are necessary in order to make Theorem 2 remain true. Let $M=D \times(\mathbf{C}-\{-1,1\}) \subset P_{1}(\mathbf{C}) \times P_{1}(\mathbf{C})$. Since both $D$ and $\mathbf{C}-\{-1,1\}$ are hyperbolically imbedded in $P_{1}(C)$, example (7) of $\$ 2$ implies that $M$ is hyperbolically imbedded in $P_{1}(\mathbf{C}) \times P_{1}(\mathbf{C})$. Let $X=D \times D$ and $A=\{(z, w) \mid z=0$ or $z= \pm w\}$ and define $f: X-A \rightarrow M$ by $f(z, w)=(z, w / z)$. Clearly $f$ does not extend to all of $X$, since $f(0,0)$ cannot be defined in a continuous way.

This example is more or less typical of the general situation. To see this consider the following generalization. Let $A$ be a closed complex subspace of $X=D^{n}$ $=D \times \cdots \times D$ with $(0, \cdots, 0) \in A$. Let $L$ denote the tautological line bundle over 
$P_{n-1}(\mathrm{C})$ (i.e. $L$ is the line bundle associated to the principal $\mathrm{C}^{*}$ bundle $\pi: \mathrm{C}^{n}-$ $\{(0, \cdots, 0)\} \rightarrow P_{n-1}(\mathrm{C})$ where $\pi$ is the natural projection). Then $X-A$ can be considered as a subspace of $L$. If $X-A$ is hyperbolically imbedded in $L$, then the inclusion $i: X-A \rightarrow L$ is a counterexample to the singular version of Theorem 2. Note that in these examples the maps do extend to a meromorphic map. This is always the case.

Theorem 3. Let $A$ be a closed complex subspace of a complex space $X$ and let $M$ be byperbolically imbedded in $Y$. If $f: X-A \rightarrow M$ is bolomorpbic, then $f$ extends to a meromorphic map $f: X \rightarrow Y$.

Proof. By a resolution of $A$ in $X$ we shall mean a triple $(Z, B, \phi)$ where

(i) $Z$ is a nonsingular complex manifold (not necessarily connected).

(ii) $B$ is a complex subspace of $Z$ whose singularities are normal crossings.

(iii) $\phi: Z \rightarrow X$ is a proper holomorphic map onto $X$ with $\phi^{-1}(A)=B$ and such that $\phi^{-1}$ is meromorphic. By Hironaka [2], such a resolution always exists (at least locally). Define $\bar{f}: Z-B \rightarrow M$ by $\bar{f}=f \circ \phi$. By Theorem $2, \bar{f}$ extends to a holomorphic map $\bar{f}: Z \rightarrow Y$. Thus $f$ extends meromorphically to $X$ by defining $f=\bar{f} \circ \phi^{-1}$. Q.E.D.

We shall now weaken the restrictions on the singularities of $A$ given in Theorem 2.

Theorem 4. Let $A$ be a closed complex subspace of a normal complex space $X$. Assume that $(Z, B, \phi)$ is a resolution of $A$ in $X$ (see proof above) such that for every pair of points $p$ and $q$ in $B$ with $\phi(p)=\phi(q)$, there exist sequences $\left\{p_{n}\right\}$ and $\left\{q_{n}\right\}$ in $Z-B$ with $p_{n} \rightarrow p, q_{n} \rightarrow q$ and $d_{X-A}\left(\phi\left(p_{n}\right), \phi\left(q_{n}\right)\right) \rightarrow 0$. If $M$ is byperbolically imbedded in $Y$, then any bolomorphic map $f: X-A \rightarrow M$ extends to a bolomorpbic map $f: X \rightarrow Y$.

Proof. Let $\bar{f}: Z \rightarrow Y$ be defined as in the last proof. Since $X$ is normal, it suffices to show that the meromorphic map $f=\bar{f} \circ \phi^{-1}$ is single valued. Since $M$ is hyperbolically imbedded in $Y$ and since $\left.f\right|_{X-A}$ is distance decreasing, we have

$$
\bar{f}(p)=\lim _{n \rightarrow \infty} f\left(\phi\left(p_{n}\right)\right)=\lim _{n \rightarrow \infty} f\left(\phi\left(q_{n}\right)\right)=\bar{f}(q) .
$$

This implies that $f$ is single valued. Q.E.D.

Remark. If $X$ is nonsingular and if $A$ has only normal crossings, then $A$ obviously satisfies the conditions of Theorem 4. Thus Theorem 4 is a generalization of Theorem 2, but in practice it is not a very satisfactory one because we are usually unable to actually construct a resolution. However, it does not seem very easy to give a condition which depends only on $X-A$ and which is not too restrictive. 
In our final generalization of the Picard theorem, we consider the space $M_{n}=\left\{\left(w_{1}, \cdots, w_{n}\right) \in \mathbf{C}^{n} \mid w_{i} \neq 0\right.$ and $w_{i} \neq 1$ for $\left.i=1, \cdots, n\right\}$. Let $\left[\left(w_{1}, v_{1}\right)\right.$, $\left.\cdots,\left(w_{n}, v_{n}\right)\right]$ be "homogeneous" coordinates for $P_{1}(\mathbf{C}) \times \cdots \times P_{1}(\mathrm{C})$ and let $\left(w_{0}, w_{1}, \cdots, w_{n}\right)$ be homogeneous coordinates for $P_{n}(\mathrm{C})$. Define $\phi: \mathrm{C}^{n} \rightarrow$ $P_{1}(\mathbf{C}) \times \cdots \times P_{1}(\mathbf{C})$ by $\phi\left(w_{1}, \cdots, w_{n}\right)=\left[\left(w_{1}, 1\right), \ldots,\left(w_{n}, 1\right)\right]$ and define $\psi$ : $\mathbf{C}^{n} \rightarrow P_{n}(\mathbf{C})$ by $\psi\left(w_{1}, \cdots, w_{n}\right)=\left(1, w_{1}, \cdots, w_{n}\right)$. Thus $\phi$ imbeds $M_{n}$ in $P_{1}(\mathrm{C}) \times \cdots \times P_{1}(\mathrm{C})$ and $\psi$ imbeds $M_{n}$ in $P_{n}(\mathrm{C})$. By (7) of $\$ 2, M_{n}$ is hyperbolically imbedded in $P_{1}(\mathrm{C}) \times \cdots \times P_{1}(\mathrm{C})$ and therefore the results of this section apply in this case. On the other hand, an easy computation shows that the identity map $i: M_{n} \rightarrow M_{n}$ does not extend to a holomorphic map $i: P_{1}(C) \times \cdots \times P_{1}(C)$ $\rightarrow P_{n}(\mathrm{C})$. Since the singularities of $A=P_{1}(\mathrm{C}) \times \cdots \times P_{1}(\mathrm{C})-M_{n}$ are normal crossings, Theorem 2 implies that $M_{n}$ is not hyperbolically imbedded in $P_{n}(\mathrm{C})$. However, we still have

Theorem 5. Let $A$ be a closed submanifold of a complex manifold $X$. Then every bolomorpbic map $f: X-A \rightarrow M_{n}$ extends to a bolomorpbic map $f: X \rightarrow P_{n}(\mathbf{C})$.

Proof. By Theorem 2, $f$ extends to a holomorphic map $f: X \rightarrow P_{1}(C) \times \cdots$ $\times P_{1}(\mathrm{C})$. We can assume $X=D^{m}=\left\{\left(z_{1}, \ldots, z_{m}\right) \in \mathrm{C}^{m}|| z_{i} \mid<1\right.$ for $\left.i=1, \ldots, m\right\}$ and that $A=\left\{\left(z_{1}, \cdots, z_{m}\right) \in D^{m} \mid z_{1}=0\right\}$. Let $f=\left(f_{1}, \cdots, f_{n}\right)$ where $f_{i}: X \rightarrow$ $P_{1}(\mathrm{C})$. Without loss of generality, we can assume that $f_{i}$ is given by two holomorphic functions, i.e. $f_{i}\left(z_{1}, \cdots, z_{n}\right)=\left(g_{i}\left(z_{1}, \cdots, z_{n}\right), b_{i}\left(z_{1}, \cdots, z_{n}\right)\right)$. Since $\left.b_{i}\right|_{X-A} \neq 0$, we see that either $\left.b_{i}\right|_{A} \equiv 0$ or $\left.b_{i}\right|_{A} \neq 0$. This implies that $b_{i}\left(z_{1}, \cdots, z_{n}\right)=z_{1}^{k_{i}} \hat{b}_{i}\left(z_{1}, \cdots, z_{n}\right)$ where $k_{i} \geq 0$ and $\hat{b}_{i}\left(z, \cdots, z_{n}\right) \neq 0$ in $X$. By the same reasoning, we have $g_{i}\left(z_{1}, \cdots, z_{n}\right)=z_{1}^{k_{i}^{\prime}} \hat{g}_{i}\left(z_{1}, \ldots, z_{n}\right)$ where $\hat{g}_{i}\left(z_{1}, \cdots, z_{n}\right) \neq 0$ in $X$. This means that $f($ i.e. $\psi \circ f)$ is given by

$$
f\left(z_{1}, \cdots, z_{n}\right)=\left(1, z_{1}^{l} \frac{\hat{g}_{1}\left(z_{1}, \cdots, z_{n}\right)}{\hat{h}_{1}\left(z_{1}, \cdots, z_{n}\right)}, \ldots, z_{1}^{l} \frac{\hat{g}_{n}\left(z_{1}, \ldots, z_{n}\right)}{\hat{h}_{n}\left(z_{1}, \ldots, z_{n}\right)}\right)
$$

where $l_{i}=k_{i}^{\prime}-k_{i}$. This implies that $f$ extends to a holomorphic map $f: X \rightarrow$ $P_{n}(\mathrm{C})$. Q.E.D.

4. Applications. Let $\mathfrak{D}$ be a bounded symmetric domain in $\mathbf{C}^{n}$ and let $\Gamma$ be an arithmetically defined, discrete subgroup of the largest connected group of holomorphic automorphisms of $\mathfrak{D}$. Assume $\Gamma$ acts freely on $\mathscr{D}$. There are two compactifications of $\mathfrak{D} / \Gamma$. The Satake compactification $(\mathcal{D} / \Gamma)_{\mathrm{B} . \mathrm{B}}^{*}$ is the same as that of Borel-Baily as a topological space. The other compactification $(\mathscr{D} / \Gamma)_{\mathrm{P} . \mathrm{s}}^{*}$ is that of PyateckiY-Šapiro. In an unpublished work, Borel proved Corollary 1 for $(\mathscr{D} / \Gamma)_{B \text {. B. }}^{*}$ His proof shows that $\mathscr{D} / \Gamma$ is hyperbolically imbedded in

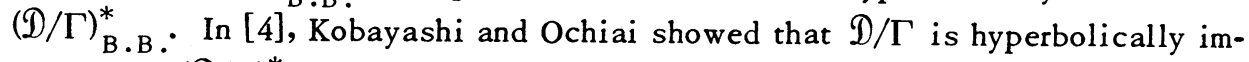
bedded in $(\mathscr{D} / \Gamma)_{\mathrm{P} . \mathrm{s}}^{*}$ and proved Corollary 1 for this case when $A$ is nonsingular. Baily has shown that there is a one-one continuous map $\psi:(\mathscr{D} / \Gamma)_{\mathrm{B} . \mathrm{B} .}^{*} \rightarrow(\mathfrak{D} / \Gamma)_{\mathrm{P} . \mathrm{s} .}^{*}$, 


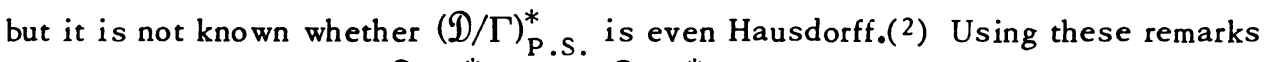

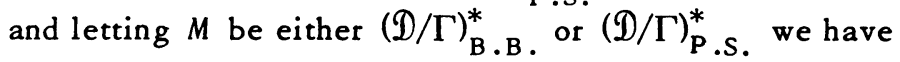

Corollary 1. Let $X$ and $A$ be as in Theorem 4 (or 2). Then any bolomorphic map $f: X-A \rightarrow \mathscr{D} / \Gamma$ extends to a bolomorpbic map $f: X \rightarrow M$. If $A$ is a complex subspace of a complex space $X$, then any bolomorphic map $f: X-A \rightarrow \mathscr{D} / \Gamma$ extends to a meromorphic map $f: X \rightarrow M$. In particular, if $f: \mathscr{D} / \Gamma \rightarrow \mathscr{D}^{\prime} / \Gamma^{\prime}$ is bolomorphic, then $f$ extends to a meromorphic map $f: M \rightarrow M$ '.

The next corollary can be interpreted as a "generalization" of Liouville's theorem.

Corollary 2. Let $X$ be a complex manifold with $d_{X} \equiv 0$ and let $A$ be a closed complex subspace of $X$. If $M$ is a compact byperbolic space and $f: X-A \rightarrow M$ is bolomorphic, then $f$ is a constant map. (For example, any map from $\mathbf{C}-\{0,1\}$ into a compact Riemann surface of genus $\geq 2$ is a constant map.) If $X-A$ is byperbolic, and $\Gamma$ is a group of automorphisms of $X-A$ such that $X-A$ is a covering space of $(X-A) / \Gamma$, then $(X-A) / \Gamma$ is not compact.

Proof. Since $M$ is compact hyperbolic, $f$ extends to a map $f: X \rightarrow M$. Since $d_{X} \equiv 0$ and $d_{M}$ is a proper distance, the distance decreasing property of $f$ implies that $f$ is a constant map. Q.E.D.

Corollary 3. Let $V \subset P_{n}(\mathrm{C})$ be a complex manifold and let $M_{n} \subset P_{n}(\mathrm{C})$ be as in Theorem 5. Let $M=V \cap M_{n^{*}}$ Let $A$ be a closed complex subspace of a complex space $X$. Then any holomorphic map $f: X-A \rightarrow M$ extends to a meromorphic map $f:$ $X \rightarrow V$. If $X$ and $A$ are both nonsingular, then $f: X \rightarrow V$ is bolomorphic.

Proof. Since $M$ is hyperbolically imbedded in $P_{1}(C) \times \cdots \times P_{1}(C)$, it is clear that $f$ extends meromorphically. The second statement follows directly from Theorem 5. Q.E.D.

The previous corollary generalizes Corollary A of a recent paper of Griffiths [1]. Griffiths has shown that under the proper restrictions on the imbedding of $V$ in $P_{n}(\mathrm{C}), M$ will have the property that it is covered by a bounded domain in $\mathrm{C}^{m}$ which is topologically a cell.

5. Conclusion. In this paper we have considered generalizations of the big Picard theorem. Except for Theorem 5, we have replaced the range space $P_{1}(C)$ $\{3$ points\} by a hyperbolically imbedded complex space, and then we have tried to replace the domain space $D^{*}$ by more general spaces. Now we comment on the relevency of the assumption "hyperbolically imbedded".

To do this consider the following examples.

(i) Let $M^{\prime}=\left\{\left(z_{0}, z_{1}, z_{2}\right) \in P_{2}(\mathrm{C}) \mid z_{0} \neq 0, z_{1} \neq 0, z_{1} \neq z_{0}, z_{2} \neq \pm z_{0} e^{z_{0} / z_{1}}\right\}$.

(2) Borel has recently shown that the two compactifications of $D / \Gamma$ are equivalent. 
Define $\phi: M^{\prime} \rightarrow(\mathrm{C}-\{0,1\}) \times(\mathrm{C}-\{-1,1\})$ by

$$
\phi\left(z_{0}, z_{1}, z_{2}\right)=\left(z_{1} / z_{0},\left(z_{2} / z_{0}\right) e^{-z_{0} / z_{1}}\right) \text {. }
$$

Clearly $\phi$ is a biholomorphism and thus $M^{\prime}$ is complete hyperbolic. However, the map $g: D^{*} \rightarrow M^{\prime}$ defined by $g(z)=\left(1, z, 2 e^{1 / z}\right)$ does not extend to a meromorphic map $g: D \rightarrow P_{2}(\mathbf{C})$.

(ii) Let $M^{\prime \prime}=\left\{\left(z_{0}, z_{1}, z_{2}\right) \in P_{2}(\mathrm{C}) \mid z_{0} \neq 0, z_{1} \neq 0, z_{1} \neq z_{0}, z_{2} z_{1} \neq \pm z_{0}^{2}\right\}$. Since the map $b: D^{*} \times D \rightarrow M^{\prime \prime}$ defined by $b(z, w)=(1, z, w / z)$ does not extend to a holomorphic map $b: D \times D \rightarrow P_{2}(C), M^{\prime \prime}$ is not hyperbolically imbedded in $P_{2}(\mathrm{C})$. Define $\psi: M^{\prime \prime} \rightarrow(\mathrm{C}-\{0,1\}) \times(\mathrm{C}-\{-1,1\})$ by $\psi\left(z_{0}, z_{1}, z_{2}\right)=$ $\left(z_{1} / z_{0}, z_{1} z_{2} / z_{0}^{2}\right)$. It is easy to see that $\psi$ is a biholomorphism which extends to a bimeromorphism $\psi: P_{2}(\mathrm{C}) \rightarrow P_{1}(\mathrm{C}) \times P_{1}(\mathrm{C})$. Thus Theorem 3 holds for maps into $M "$.

The first example shows that some assumptions on the way in which $M$ is contained in $Y$ are necessary if any extension theorem is going to work. The second example indicates that it is not necessary for $M$ to be hyperbolically imbedded in $Y$ in order for Theorem 3 to be true. In fact, it is possible that it may be sufficient for $M$ to be a (complete) hyperbolic Zariski open set of a closed algebraic manifold $Y$. It also shows that these last conditions are not strong enough to imply that you can extend holomorphically over a nonsingular subspace. Theorem 5 implies that "hyperbolically imbedded" is not a necessary condition for the nonsingular version of Theorem 2. Finally, the discussion preceding Theorem 5 indicates that strong assumptions such as "hyperbolically imbedded" are probably necessary if Theorem 2 is to hold.

\section{REFERENCES}

1. P. A. Griffiths, Complex-analytic properties of certain Zariski open sets on algebraic varieties, Ann. of Math. (2) 94 (1971), 21-51.

2. H. Hironaka, Resolution of singularities of an algebraic variety over a field of characteristic zero. I, II, Ann. of Math (2) 79 (1964), 109-326. MR 33 \#7333.

3. S. Kobayashi, Hyperbolic manifolds and holomorphic mappings, Pure and Appl. Math., no. 2, Dekker, New York, 1970. MR 43 \#3503.

4. S. Kobaya shi and T. Ochiai, Satake compactifications and the great Picard theorem, J. Math. Soc. Japan 23 (1971), 340-350.

5. M. H. Kwack, Generalization of the big Picard theorem, Ann. of Math. (2) 90 (1969), 9-22. MR 39 \#4445.

6. R. Remmert, Holomorphe und meromorphe Abbildungen komplexer Räume, Math. Ann. 133 (1957), 328-370. MR 19, 1193.

DEPARTMENT OF MATHEMATICS, UNIVERSITY OF BRITISH COLUMBIA, VANCOUVER 8, B.C., CANADA 